\title{
Accelerated cortical \\ thinning correlates with early signs of depression
}

\author{
By Dr Jessica K Edwards
}

The brain undergoes structural changes as it develops over childhood, but whether abnormal structural changes are associated with emerging depressive symptoms in adolescence is unknown. Now, a longitudinal study that enrolled 205 participants aged 8-25 years without signs of depression has used magnetic resonance imaging (MRI) to monitor these brain changes over adolescence. The participants underwent MRI every 2 years over a 5 -year period to monitor changes in cortical thickness, surface area and sub-cortical volume. At the third MRI, depressive symptoms were assessed by self-report (Beck Depression Inventory II). Those who had developed depressive symptoms by this time point, showed accelerated frontal lobe cortical thinning compared to those without depressive symptoms. Interestingly, changes in hippocampal and amygdala volume were not associated with depressive symptoms, despite previous studies suggesting a potential link between volumetric differences in these brain regions and depression. The researchers also noted that accelerated cortical thinning in adolescence has previously been associated with positive behavioural outcomes, such as improved cognitive performance ${ }^{2}$, yet here it was associated with an adverse outcome. The researchers propose that accelerated frontal lobe cortical thinning may be associated with emerging depressive symptoms in adolescence.

Bos, M.G.N., Peters, S., van de Kamp, F.C., Crone, E.A. \& Tamnes, C.K. (2018), Emerging depression in adolescence coincides with accelerated frontal cortical thinning. J Child Psychol Psychiatr. doi:10.1111/jcpp.12895

Further reading:

${ }^{1}$ Schmaal, L. et al. (2016). Subcortical brain alterations in major depressive disorder: Findings from the ENIGMA Major Depressive Disorder working group. Molecular Psychiatry, 21, 806-812. doi:10.1038/mp.2015.69

${ }^{2}$ Shaw, P. et al. (2006). Intellectual ability and cortical development in children and adolescents. Nature 440:676-679. doi: 10.1038/nature04513

Glossary:

Magnetic resonance imaging: a non-invasive technique that uses a strong, static magnetic field and radio waves to measure brain activity

Beck Depression Inventory II (BDI-II): The BDI-II (1996) is a revision of the original $B D I(1961)$ in response to changes in the DSM-IV criteria for major depressive disorder. BDI-II measures depression symptoms and severity in adolescents aged $\geq 13$ years over a 2-week time-frame, through answers to 21 multiple choice questions

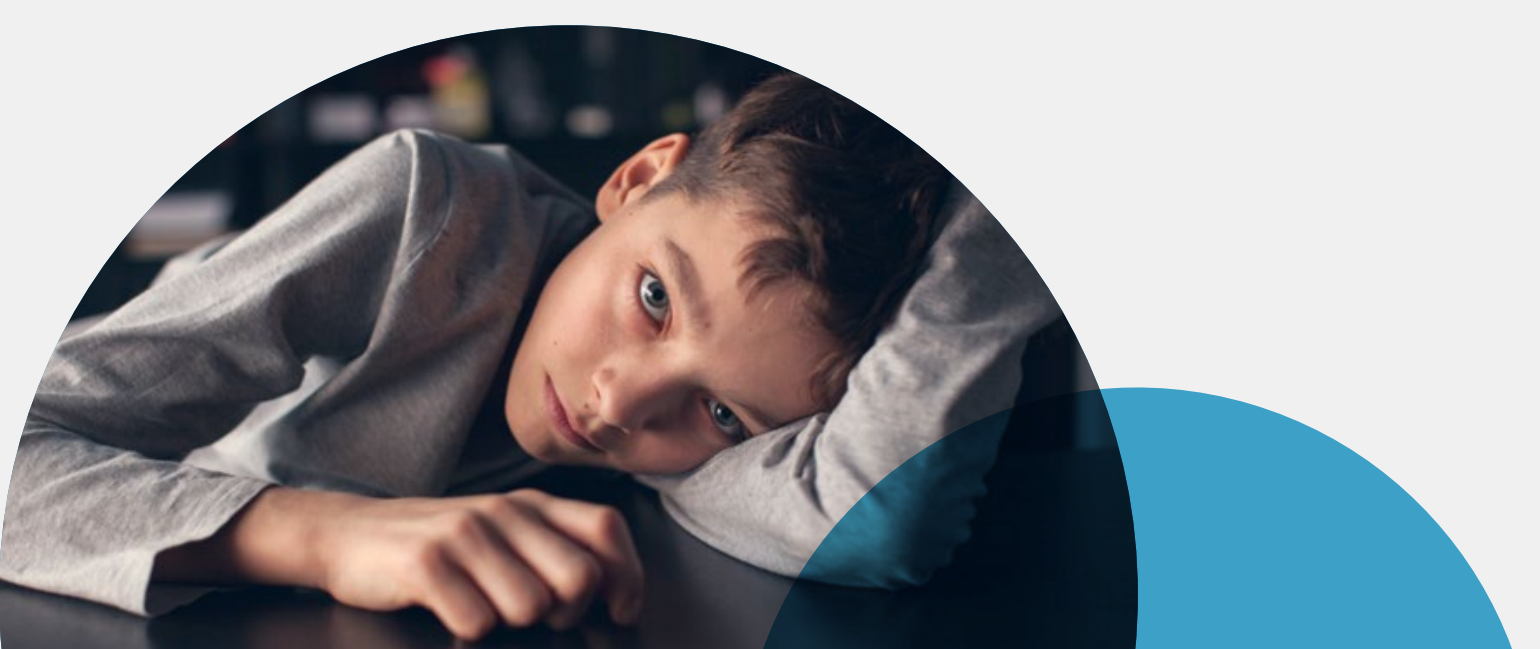

